\section{Interventions to improve thyroid cancer survivors' quality of life}
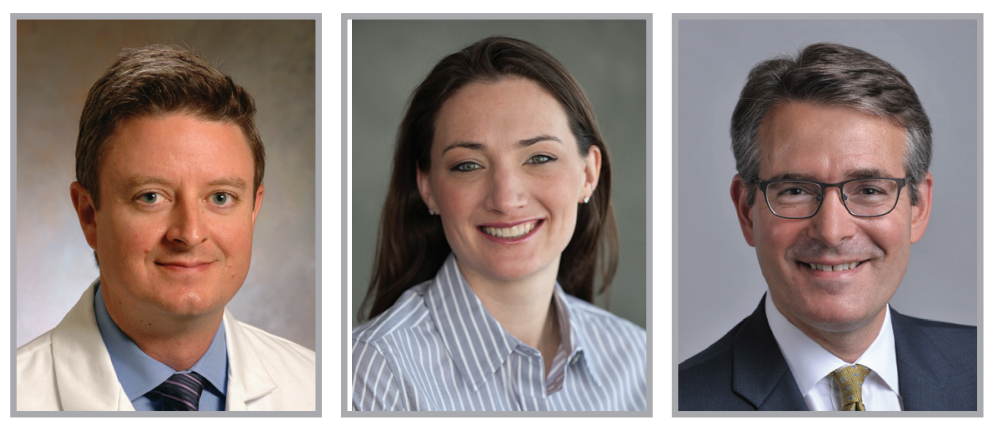

Raymon H Grogan*,1, Briseis Aschebrook-Kilfoy ${ }^{2} \&$ Peter Angelos ${ }^{1}$

First draft submitted: 25 January 2016; Accepted for publication: 2 March 2016; Published online: 7 April 2016

Interventions to improve thyroid cancer survivors' quality of life (QoL) have been a topic of interest for our research group for some time now, at least since December of 2013, the start of the NATCSS study [1]. At the time, we wanted to know how thyroid cancer affects QoL of survivors. After an extensive literature review, we still did not have an answer, and thus the NATCSS was born. The analysis of the first 1000 participants was completed at the end of 2015, and from it we found that thyroid cancer has a detrimental impact on a person's QoL, and the effect is much larger than anyone anticipated [1]. In fact, QoL in thyroid cancer survivors is about the same or worse than breast and colorectal cancer survivors [2]. Of course it's one thing to know something, it's an entirely different thing to try and fix it. A PubMed search on studies of intervention strategies for QoL in cancer survivors is revealing. The simple, informal PubMed query "...cancer quality of life intervention" is a look into how society, or at the minimum the medical research community, views the relative importance of cancer survivorship care among various cancer types. Inserting 'breast' into this query returns 1014 results, 'prostate' returns 425, 'lung' returns 332, 'colorectal' returns 289, 'ovarian' returns 99 and 'thyroid' returns 39. It is striking to note that this trend exactly mirrors the National Cancer Institute's research funding rates for these cancers [3].

As far as we can tell, a more detailed search for studies of interventions to improve QoL in thyroid cancer survivors shows that there are approximately zero studies on this subject. Realistically, the only evidence-based answer to the question of how to improve QoL in thyroid cancer is: 'no one knows'. It reasonably follows then that if we want to make interventions based on evidence, we as a medical/scientific community have to take the time to study intervention strategies for thyroid cancer survivors. This also means

\section{KEYWORDS}

- quality of life intervention

- survivorship quality of life $\bullet$ thyroid cancer $\bullet$ thyroid cancer survivors

“...we wanted to know how thyroid cancer affects quality of life of survivors. After an extensive literature review, we still did not have an answer, and thus the NATCSS was born.” 


\section{"We propose the development of a short- form questionnaire ... We also propose that the tool be incorporated into all thyroid cancer survivorship care plans on a national level.”}

that NIH funding for thyroid cancer needs to be increased, but we digress, as that is the subject of an entirely different editorial [3].

What we do have is a good baseline understanding of the key issues that may arise for thyroid cancer survivors (based on findings from NATCSS) [1]. Until there are studies testing specific thyroid cancer QoL interventions, we will be forced to draw inferences of the efficacy of various intervention strategies from studies in other cancer survivor groups. The danger with this approach is that findings from studies of other cancer types, such as breast cancer survivors, are not translatable to thyroid cancer survivors. However, based on the NATCSS findings and intervention strategies applied to other cancer types, here are some suggestions for ways to improve QoL in thyroid cancer survivors.

The first two types of intervention strategies are rooted in education. The idea for these two interventions are based on data from NATCSS and we believe may have a significant positive impact on QoL in thyroid cancer survivors.

\section{Eliminate the phrase 'thyroid cancer is the good cancer'}

We recently polled a group of 105 surgeons and endocrinologists who specialize in thyroid cancer care and found that $54 \%$ of them routinely tell their patient that this is 'the good cancer' (data under review). An increased awareness of the true long-term prognosis of thyroid cancer needs to be more clearly communicated and understood by both patient and physician. Yes it is true that the long-term survival for thyroid cancer is better than for most other cancers $(3 \%$ of thyroid cancer patients will die within 5 years of their original diagnosis), but that does not make it 'good' [4]. We cannot think of any other disease state in which physicians congratulate the patient on having contracted an illness. While the use of the word 'good' in this context may seem like a matter of semantics, take into consideration that up to $30 \%$ of patients may have a recurrence that necessitates further surgery and radioiodine therapy later in life [5]. When a patient has a recurrence after being disease free for several years we find in our own clinic a common patient reaction is to have a sense of bewilderment, frustration, sadness, and sometimes anger because they were told by their doctor that this was a 'good' cancer. Often these same patients had been told that they need never worry about their thyroid cancer again, yet here they are getting ready to go through another round of surgery and radioiodine therapy. This sentiment is corroborated by NATCSS findings in which up to $30 \%$ of thyroid cancer survivors feel that their cancer, and/or side effects from treatment are not taken seriously by either their physicians or their family members [1]. A more appropriate expectation to convey to a newly diagnosed thyroid cancer patient is that yes the survival rates are high, but that does not mean that after the initial therapy there is a guarantee of complete 'cure'. Unfortunately, there is no foolproof method to distinguish those that will have future problems with the cancer from those that will not. While current staging systems are useful as rough guidelines on a population level, they cannot be used to individualize prediction of outcomes for any one patient. This is a known limitation of all cancer staging guidelines, but is particularly problematic in thyroid cancer as evidenced by the fact that there are over 17 different staging guidelines described in the literature [6]. By setting appropriate expectations on outcomes, the patient's understanding should be better aligned with the reality of thyroid cancer, as well as with the physicians understanding and expectation of the disease. This should allow for a faster and healthier psychological adjustment to life after treatment for survivors. Mismatch in expectations between the physician and patient is a form of miscommunication that we believe has significant psychological consequences for those patients who do recur or face death from their disease [7].

\section{Better education on QoL issues that may arise for thyroid cancer survivors}

With findings from the NATCSS, we now know that some thyroid cancer survivors will have significant short- and/or long-term struggles with QoL. We also have an idea of which patients are at higher risk for possible QoL issues as well as specific areas of life in which thyroid cancer survivors struggle. For example, the NATCSS data show that the three biggest risk factors for decreased QoL is young age, female gender and low education attainment. Additionally the psychological QoL subscores were lower than the other three subdomains tested, namely physical, social and spiritual [1]. It is important that physicians have a frank conversation with survivors at some point, so that if an issue arises the patient does not feel minimized or as if they have no one to talk to them about these issues. 


\section{Develop a short, clinically useful tool to} assess QoL in thyroid cancer survivors

QoL issues are often times not readily apparent in a routine clinic visit. It is not as simple as asking a patient, "so how do you feel today?" While this question is a reasonable start, the fact is that survivors themselves might not recognize that they are having difficulties. It is also known that patients may not want to bring up issues that they think the physician might deem trivial or inconsequential. There are many factors that make it difficult to evaluate QoL in the clinic. However, it has been shown in other cancer survivors that simple, validated questionnaires that take only minutes to fill out can be very accurate at identifying those who are having difficulties coping with or adjusting to their life after a cancer diagnosis [8]. Such a tool currently does not exist for thyroid cancer survivors. The tool used for the NATCSS study was a questionnaire of nearly 75 questions. Unfortunately, a questionnaire of this length may not be a clinically useful tool. We propose the development of a short-form questionnaire. It should be simple, 20 questions at the most, easily administered at any time and anywhere, with an easily identifiable QoL score at the end of the assessment. We also propose that the tool be incorporated into all thyroid cancer survivorship care plans on a national level.

\section{Prospective trials using intervention strategies known to work in other cancer survivors}

There are several types of intervention strategies that have been tried in many different cancer survivor groups. Some intervention strategies that have been used in other cancer survivor groups include education, social support development, cognitive-behavioral techniques, formal counseling, financial counseling and exercise and nutrition interventions [9]. There are likely many others that we have not listed. Due to disease differences, and basic demographics, not every intervention strategy works the same in the different cancer groups. If improving education on QoL in thyroid cancer survivors shows no improvement in QoL then its utility as an intervention is suspect. But we will not know if this is true without asking the question and seeking the answer.

The bottom line is that the question of what interventions can improve the QoL of thyroid cancer survivors can no longer be ignored. We now know that thyroid cancer survivors face significant challenges, and it is important that we as a medical community do what we can to alleviate these problems. Our work in this area has started to bring to light the myriad of issues reported over and over by survivors. Without investigation into potential interventions to improve QoL, we will continue to have struggling patients for whom we do not have answers.

\section{Financial \& competing interests disclosure}

The authors have no relevant affliations or financial involvement with any organization or entity with a financial interest in or financial conflict with the subject matter or materials discussed in the manuscript. This includes employment, consultancies, honoraria, stock ownership or options, expert testimony, grants or patents received or pending, or royalties.

No writing assistance was utilized in the production of this manuscript.

\section{References}

1 Aschebrook-Kilfoy B, James B, Nagar S et al. Risk factors for decreased quality of life in thyroid cancer survivors: initial findings from the North American Thyroid Cancer Survivorship Study. Thyroid 25(12), 1313-1321 (2015).

2 Applewhite MK, James BC, Kaplan SP et al. Quality of life in thyroid cancer is similar to that of other cancers with worse survival. World J. Surg. 40 (3), 551-561 (2015).

3 Aschebrook-Kilfoy B, Schechter RB, Shih Y-CT et al. The clinical and economic burden of a sustained increase in thyroid cancer incidence. Cancer Epidemiol. Biomarkers Prev. 22(7), 1252-1259 (2013).

4 Davies L, Welch HG. Increasing incidence of thyroid cancer in the United States, 1973-2002. JAMA 295(18), 2164-2167 (2006).

5 Grogan RH, Kaplan SP, Cao H et al. A study of recurrence and death from papillary thyroid cancer with 27 years of median follow-up. Surgery 154(6), 1436-1447 (2013).

6 Lang BH, Lo CY, Chan WF, Lam KY, Wan KY. Staging systems for papillary thyroid carcinoma: a review and comparison. Ann. Surg. 245(3), 366-378 (2007).
7 Politi MC, Studts JL, Hayslip JW. Shared decision making in oncology practice: what do oncologists need to know? Oncologist 17(1), 91-100 (2012).

8 Chopra I, Kamal KM. A systematic review of quality of life instruments in long-term breast cancer survivors. Health Qual. Life Outcomes 10, 14 (2012).

9 Hersch J, Juraskova I, Price M, Mullan B. Psychosocial interventions and quality of life in gynaecological cancer patients: a systematic review. Psychooncology 18(8), 795-810 (2009). 